\title{
Lifetime risk of stroke and impact of hypertension: estimates from the adult health study in Hiroshima and Nagasaki
}

\author{
Ikuno Takahashi ${ }^{1,4}$, Susan M Geyer ${ }^{2,6}$, Nobuo Nishi ${ }^{3,7}$, Tomohiko Ohshita ${ }^{4}$, Tetsuya Takahashi ${ }^{4}$, \\ Masazumi Akahoshi ${ }^{1}$, Saeko Fujiwara ${ }^{1}$, Kazunori Kodama ${ }^{5}$ and Masayasu Matsumoto ${ }^{4}$
}

Very few reports have been published on lifetime risk (LTR) of stroke by blood pressure (BP) group. This study included participants in the Radiation Effects Research Foundation Adult Health Study who have been followed up by biennial health examinations since 1958. We calculated the LTR of stroke for various BP-based groups among 7847 subjects who had not been diagnosed with stroke before the index age of 55 years using cumulative incidence analysis adjusting for competing risks. By 2003,868 subjects had suffered stroke $(512(58.9 \%)$ were women and $542(62.4 \%)$ experienced ischemic stroke). BP was a significant factor in determining risk of stroke for men and women, with distributions of cumulative risk for stroke significantly different across BP groups. The LTR of all-stroke for normotension (systolic BP/diastolic BP $<120 / 80 \mathrm{~mm} \mathrm{Hg}$ ), prehypertension (120-139/80-89 mm Hg), stage 1 hypertension (140-159/90-99 mm Hg) and stage 2 hypertension ( $>160 / 100 \mathrm{~mm} \mathrm{Hg}$ ) were 13.8-16.9-25.8-25.8\% in men and 16.0-19.9-24.0-30.5\% in women, respectively $(P<0.001$ among BP groups in both sexes). The estimates did not differ significantly $(P=0.16)$ between normotensive and prehypertensive subjects. One in five Japanese atomic bomb survivor subjects experienced stroke over their lifetime from the age of 55 years. Long-term stroke risks were elevated in those with hypertension $(>140 / 90 \mathrm{~mm} \mathrm{Hg}$ ) at any of the index ages of 45, 55, 65 and 75 years. Hypertension Research (2011) 34, 649-654; doi:10.1038/hr.2011.7; published online 17 February 2011

Keywords: blood pressure; epidemiology; lifetime risk; stroke

\section{INTRODUCTION}

Stroke mortality is higher in Japan than in other developed countries, ${ }^{1}$ and the incidence of stroke among Japanese subjects has remained high in recent years, especially in the elderly. ${ }^{2}$ With aging of the population, stroke has become an ever more important health burden in Japan, and thus activities aiming at stroke prevention require urgent attention. Elevated blood pressure (BP) has emerged as one of the prominent risk factors for stroke. ${ }^{3}$

Lifetime risk (LTR) estimates, which represent risk of the disease of interest and adjust for competing risk of death from other causes, provide a simple conceptual basis for estimating absolute risk of developing a disease during the remainder of one's life. ${ }^{4}$ Estimating the LTR in relation to known risk factors for stroke, such as hypertension, can help to highlight the magnitude and influence of these risk factors and the public health burden associated with them. Past estimates of the LTR of stroke in relation to known risk factors and the corresponding burden on the population, however, are limited. ${ }^{4-6}$ The objectives of this study were to determine the LTR of stroke and to clarify long-term effects of BP levels at midlife in a Japanese population from the Adult Health Study (AHS) of atomic bomb survivors.

\section{METHODS}

Study population

The Radiation Effects Research Foundation (RERF; formerly the Atomic Bomb Casualty Commission or ABCC) established a longitudinal Life Span Study cohort consisting of 120321 Japanese atomic bomb survivors in $1950^{7,8}$ and its subcohort, AHS, consisting of 19961 survivors in 1958. The AHS includes individuals exposed to a wide range of radiation doses; about half were within $2 \mathrm{~km}$ of the hypocenter (proximal exposure), a quarter were at distances of more than $3 \mathrm{~km}$ (distal exposure) and a quarter were not in the city at the time of the bombings. The AHS includes biennial health examinations and complete follow-up with respect to death. Of all subjects, more than $70 \%$ have continued to participate in each 2-year examination cycle.

To parallel methodologies used in other studies of the LTR, our primary interest was to evaluate risk of stroke starting at an index age of about 55 years. Because of the biennial nature of our clinical health examinations, and to be

\footnotetext{
${ }^{1}$ Department of Clinical Studies, Radiation Effects Research Foundation, Hiroshima, Japan; ${ }^{2}$ Department of Statistics, Radiation Effects Research Foundation, Hiroshima, Japan; ${ }^{3}$ Department of Epidemiology, Radiation Effects Research Foundation, Hiroshima, Japan; ${ }^{4}$ Department of Clinical Neuroscience and Therapeutics, Hiroshima University, Hiroshima, Japan; ${ }^{5}$ Radiation Effects Research Foundation, Hiroshima, Japan; ${ }^{6}$ Department of Internal Medicine, Division of Hematology, Ohio State University, Columbus, OH, USA and ${ }^{7}$ National Institute of Health and Nutrition, Tokyo, Japan

Correspondence: Dr I Takahashi, Department of Clinical Studies, Radiation Effects Research Foundation, 5-2 Hijiyama Park, Minami-ku, Hiroshima 732-0815, Japan. E-mail: iktakaha@rerf.or.jp
}

Received 18 May 2010; revised 20 November 2010; accepted 14 December 2010; published online 17 February 2011 
able to utilize BP measures at the index age, we defined index ages using 2-year intervals (for example, 55.0-56.9 years old (y.o.)). In addition to an index age of 55 y.o., we looked at similar index age intervals for the following: 45 y.o. (45.0-46.9), 65 y.o. (65.0-66.9) and 75 y.o. (75.0-76.9). As subjects needed to have an exam with BP measurements and be stroke free and alive at an index age, the number of subjects varied for corresponding index age-associated cohorts. For the 55 y.o. index age cohort, 7487 participants were identified as being stroke free and alive at the index age, and had BP measurements at the corresponding index age-associated clinical examination during the study period between 1 July 1958 and 31 December 2003. Participants were monitored until they developed their first-ever stroke, which was identified at the examination or by death certificate, died, or until their most recent RERF evaluation before December 2003, whichever came first. Informed consent was obtained from all participants, and the Ethics Committee at RERF approved this study.

To complete our surveillance of non-fatal and fatal events, diagnosis at each examination and underlying and contributing causes of deaths based on death certificates during the study periods were coded according to the International Classification of Disease (ICD) codes in the RERF database. The ICD codes of stroke-related disease are 330-332, 334, 352 and 435 (ICD-7), 333, 430-434, 436 and 438 (ICD-8), 430, 431 and 433-438 (ICD-9), and G45, I60, I61, I63-66 and I69 (excluding I698; ICD-10). All potential non-fatal stroke cases with these codes were systematically reviewed by one researcher (IT) regarding experience of stroke events, such as rapidly developed clinical signs of focal disturbance in cerebral function lasting more than $24 \mathrm{~h}$ (unless interrupted by surgery or death) without apparent cause other than vascular origin (for example, blood disease, brain tumor, or brain metastases). We did not consider the following symptoms as sole evidence of focal dysfunction: dizziness, vertigo, localized headache, blurred vision, dysarthria, impaired cognitive function (including confusion) or seizures according to the definitions of typical/atypical stroke symptoms in the World Health Organization (WHO)-initiated Monitoring of Trends and Determinants in Cardiovascular Disease (MONICA) Projects. ${ }^{9} 10$ Identified stroke cases were further classified by subtype of stroke based on participants' accepted diagnostic information (autopsy, computed tomography (CT) or magnetic resonance imaging (MRI) scan conducted outside of RERF, death certificate and clinical information centrally collected by one neurologist ${ }^{11}$ ). In addition, an external collaborating neurologist (TO) oversaw this process.

\section{Baseline examination}

Sitting BP was measured in the left arm after an adequate sedentary period. BP groups were defined using systolic BP (SBP) and diastolic BP (DBP) in accordance with criteria from the Seventh Report of the Joint National Committee on the Prevention, Detection, Evaluation and Treatment of High Blood Pressure (JNC 7) classification: ${ }^{12}$ normal, $\quad \mathrm{SBP}<120 \mathrm{~mm} \mathrm{Hg}$ and $\mathrm{DBP}<80 \mathrm{~mm} \mathrm{Hg}$; prehypertension, SBP $120-139 \mathrm{mmHg}$ and/or DBP 80-89 mm Hg; stage 1 hypertension, SBP $140-159 \mathrm{~mm} \mathrm{Hg}$ and/or DBP 90-99 $\mathrm{mm} \mathrm{Hg}$; and stage 2 hypertension, SBP $>160 \mathrm{mmHg}$ and/or DBP $>100 \mathrm{~mm} \mathrm{Hg}$. Non-fasting serum cholesterol levels were measured at the biennial examinations by Abell-Kendall method during 1958-1965, automated methods (Technicon Autoanalyzer, Technicon Instruments, Tarrytown, NY, USA) during 1965-1967 and a 7050 analyzer (Hitachi, Tokyo, Japan) from 1986 afterward. Measurements of height and weight were made at each examination. Profile of possible risk factors was shown in Table 1.

\section{Statistical analysis}

Gender-specific analyses of LTR of stroke and its subtypes (hemorrhagic vs. ischemic stroke) in the various index age cohorts were carried out using cumulative incidence analysis, adjusting for the competing risk of non-stroke death. Estimates and their associated estimated standard errors derived for the risk of stroke at 10,20, 30 and 40 years after the index age as well as the LTR were generated using the cmprsk, add-on package in $R .{ }^{13}$ This is one of the recommended tools for cumulative incidence analyses that can take into account competing events and also compare the cumulative incidence of a particular type of event among different factors or groups. ${ }^{14-16}$ Stroke incidence was analyzed by 3 different periods to address potential diagnostic differences as well as potential cultural changes in lifestyle: 1950-1977 vs. 1978-1989 vs. 1990 or later. ${ }^{17}$ Descriptive summary statistics and graphical analyses were used to assess each of the variables across all subjects as well as by gender. Differences in continuous variables between groups were assessed using two-sample $t$-test when assumptions did not hold and sufficient transformations were not possible. The $\chi^{2}$-tests were used to assess relationships between categorical variables. All statistical analyses were performed using the statistical software $\mathrm{R}$ program ( $\mathrm{R}$ version 2.11.1). ${ }^{13}$

\section{RESULTS}

\section{Subject characteristics}

The characteristics of the study cohort used in the analyses in terms of BP categories, serum total cholesterol levels and body mass index are shown in Table 1 for each of the index age cohorts. As our primary interest was evaluating LTR of stroke estimates from an index age of about 55 y.o. in order to be analogous to other similar studies, results presented here focus on that index age cohort unless indicated otherwise. Only $24.5 \%$ of subjects were classified as normotensive using the JNC 7 BP categories, and $40.7 \%$ had stage 1 or stage 2 hypertension. There were statistically significant differences in distribution of BP factor levels between men and women $(P<0.001)$, with a higher percentage of men classified as having stage 1 or 2 hypertension (45.5 vs. $38.0 \%$ in women). Women had significantly higher serum total cholesterol levels and body mass index than men $(P<0.001$ for both comparisons).The median follow-up time for event-free subjects was 16.1 years (range: 1 day-42.1 years), with 3954 subjects experiencing either stroke or death event. A total of 868 strokes (38\% cases were identified using only information from death certificates) and 3086 cases of non-stroke-related death occurred as of December 2003.

\section{Stroke subject characteristics}

Of the 868 subjects with valid documented stroke event, 512 (58.9\%) were women. Most strokes (62.4\%) were ischemic, with $26.6 \%$ (231 participants; 146 women) developing hemorrhagic stroke. A total of 38 subarachnoid hemorrhage events ( 28 women) were included in the overall category of hemorrhagic stroke. The subjects experienced stroke not otherwise specified, and these cases often had insufficient information to classify the stroke as either hemorrhagic or ischemic. Of the 868 strokes, 332 were verified by death certificate alone, 319 were diagnosed by neuroimaging outside of RERF, 26 were verified by autopsy, 147 were verified by medical records and 44 were verified through earlier identification by one neurologist. ${ }^{11}$ The median age of subjects experiencing stroke was 72.0 years (range: 55.4-98.4 years). There were significant differences in age of first stroke by stroke subtype $(P<0.001)$. The median age of first event was 69.6 years (range: 56.2-96.4 years) for hemorrhagic stroke, 71.7 years (range: 55.6-93.2 years) for stroke not otherwise specified and 73.5 years (range: 55.4-98.4 years) for ischemic stroke.

\section{LTR of stroke}

The LTR for both sexes reached similar levels at the 40-year risk estimate: one in five persons (20\%; Table 2). Significant gender differences were observed for all-stroke incidence $(P<0.001)$. When we evaluated strokes by subtype, however, gender differences were only observed for ischemic stroke $(P<0.001)$. At the ages of 65 and 75 years, risks of each stroke subtype were higher for men, but the LTR were higher for women.

We stratified subjects into groups who turned 55 years of age in the first (1958-1977), second (1978-1990) or third periods (1991+) in men. The LTR of all-stroke was significantly different by time period 
Table 1 Characteristics by index age cohort

\begin{tabular}{|c|c|c|c|c|c|}
\hline \multirow[b]{2}{*}{ Sex } & \multirow[b]{2}{*}{ Variables } & \multicolumn{4}{|c|}{ Index age (years) } \\
\hline & & 45 & 55 & 65 & 75 \\
\hline \multirow[t]{27}{*}{ Men } & $B P$ group $^{\mathrm{a}}(n)$ & & & & \\
\hline & Normal & 595 & 530 & 454 & 203 \\
\hline & PreHT & 758 & 913 & 871 & 429 \\
\hline & Stage $1 \mathrm{HT}$ & 366 & 698 & 747 & 409 \\
\hline & Stage $2 \mathrm{HT}$ & 282 & 507 & 651 & 391 \\
\hline & $D B P(m m H g)$ & & & & \\
\hline & Median (range) & $80(45-152)$ & $84(20-160)$ & $82(30-140)$ & $80(30-134)$ \\
\hline & $S B P(m m ~ H g)$ & & & & \\
\hline & Median (range) & $120(80-244)$ & $130(80-260)$ & $138(80-240)$ & $140(70-260)$ \\
\hline & $B M I\left(\mathrm{~kg} \mathrm{~m}^{-2}\right)$ & & & & \\
\hline & Median (range) & $21.6(12.6-34.9)$ & $21.8(12.9-38.3)$ & $22.4(12.1-37.2)$ & $21(12.7-33.4)$ \\
\hline & Total cholesterol (mg dl-1 & & & & \\
\hline & Median (range) & $174(74-361)$ & $179(75-415)$ & $179(76-354)$ & $179(80-324)$ \\
\hline & Index age time period ( $n)$ & & & & \\
\hline & 1950-1977 & 1377 & 1152 & 1188 & 474 \\
\hline & 1978-1989 & 570 & 1088 & 528 & 516 \\
\hline & 1990-2003 & 54 & 408 & 1007 & 442 \\
\hline & Strokes (n) & & & & \\
\hline & All strokes & 221 & 356 & 344 & 181 \\
\hline & Hemorrhagic stroke & 59 & 85 & 73 & 40 \\
\hline & Ischemic stroke & 146 & 238 & 227 & 124 \\
\hline & Stroke NOS & 16 & 33 & 44 & 17 \\
\hline & Non-stroke death & 866 & 1303 & 1596 & 1012 \\
\hline & Alive and stroke free & 914 & 989 & 783 & 239 \\
\hline & Follow-up from index age & & & & \\
\hline & Median (range) & $24(0.01-44.8)$ & $14.4(0.01-36.2)$ & $6.3(0.01-28)$ & $1.8(0.01-21.1)$ \\
\hline & Person-years & 41306.9 & 38061.5 & 25127.2 & 8128.6 \\
\hline \multirow[t]{17}{*}{ Women } & $B P$ group $^{\mathrm{a}}(n)$ & & & & \\
\hline & Normal & 1603 & 1306 & 963 & 379 \\
\hline & PreHT & 1324 & 1690 & 1654 & 949 \\
\hline & Stage $1 \mathrm{HT}$ & 570 & 1081 & 1267 & 928 \\
\hline & Stage $2 \mathrm{HT}$ & 289 & 762 & 1024 & 822 \\
\hline & $D B P$ & & & & \\
\hline & Median (range) & $78(40-150)$ & $80(20-180)$ & $80(8-160)$ & $80(30-160)$ \\
\hline & $S B P$ & & & & \\
\hline & Median (range) & $120(78-262)$ & $128(80-280)$ & $134(76-260)$ & $142(86-260)$ \\
\hline & $B M I\left(\mathrm{~kg} \mathrm{~m}^{-2}\right)$ & & & & \\
\hline & Median (range) & $22.4(15-42.7)$ & $22.6(14.3-42.7)$ & $22.4(12.7-42)$ & $22.3(11.8-40.2)$ \\
\hline & Total cholesterol (mg d/ & & & & \\
\hline & Median (range) & $172(85-361)$ & $199(94-426)$ & $191(90-536)$ & $202(80-409)$ \\
\hline & Index age time period ( $n$ ) & & & & \\
\hline & 1950-1977 & 2921 & 2051 & 1727 & 743 \\
\hline & 1978-1989 & 796 & 2218 & 1309 & 896 \\
\hline & 1990-2003 & 69 & 570 & 1872 & 1439 \\
\hline
\end{tabular}


Table 1 (Continued)

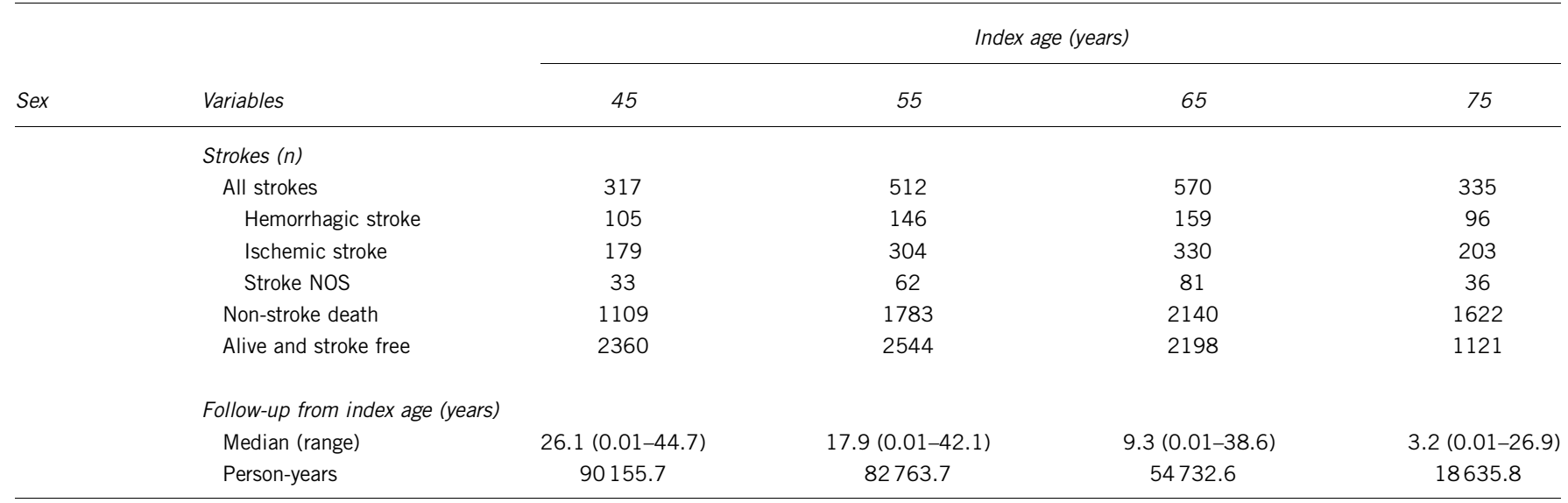

Abbreviations: BMI, body mass index; BP, blood pressure; DBP, diastolic BP; HT, hypertension; JNC 7, Seventh Report of the Joint National Committee on the Prevention, Detection, Evaluation, and Treatment of High Blood Pressure; SBP, systolic blood pressure; stroke NOS, stroke not otherwise specified.

aBP groups were defined using SBP and DBP, and followed criteria from the JNC 7 classification: ${ }^{12}$ normal, SBP $<120 \mathrm{~mm} \mathrm{Hg}$ and DBP $<80 \mathrm{~mm} \mathrm{Hg} ;$ preHT, SBP $120-139 \mathrm{~mm} \mathrm{Hg}$ and/or DBP $80-89 \mathrm{~mm} \mathrm{Hg}$; stage $1 \mathrm{HT}$, SBP $140-159 \mathrm{~mm} \mathrm{Hg}$ and/or DBP 90-99 mm Hg; and stage $2 \mathrm{HT}$, SBP $\geqslant 160 \mathrm{~mm} \mathrm{Hg}$ and/or DBP $\geqslant 100 \mathrm{~mm} \mathrm{Hg}$.

Table 2 Age- and sex-specific short-, intermediate-term and LTR estimates for stroke and its subtypes: adult health study, 45-year follow-up, 1958-2003, Japan

\begin{tabular}{|c|c|c|c|c|c|c|c|}
\hline \multirow[b]{2}{*}{ Stroke type } & \multirow[b]{2}{*}{ Sex } & \multirow{2}{*}{$\begin{array}{c}\text { Index age } \\
\text { (years) }\end{array}$} & \multicolumn{4}{|c|}{ Short- and intermediate-term risk estimate (s.d.) (\%) } & \multirow{2}{*}{$\begin{array}{l}\text { LTR estimate } \\
\text { (s.d.) (\%) }\end{array}$} \\
\hline & & & 10 year & 20 year & 30 year & 40 year & \\
\hline \multirow[t]{8}{*}{ All-stroke } & Men & 45 & $1.7(0.3)$ & $6.3(0.6)$ & $12.2(0.9)$ & $17.7(1.3)$ & $19.6(1.5)$ \\
\hline & & 55 & $5.9(0.5)$ & $12.4(0.7)$ & $18.4(1.0)$ & $20.4(1.1)$ & $20.5(1.1)$ \\
\hline & & 65 & $8.3(0.6)$ & $14.9(0.8)$ & $17.4(0.9)$ & - & $17.6(0.9)$ \\
\hline & & 75 & $10.8(1.4)$ & $15.3(1.1)$ & - & - & $15.6(1.1)$ \\
\hline & Women & 45 & $1.0(0.2)$ & $3.4(0.3)$ & $8.2(0.5)$ & $15.9(1.0)$ & $18.0(1.3)$ \\
\hline & & 55 & $2.2(0.2)$ & $7.9(0.4)$ & $16.3(0.8)$ & $21.6(1.0)$ & $22.2(1.1)$ \\
\hline & & 65 & $5.6(0.4)$ & $14.6(0.7)$ & $20.2(0.8)$ & - & $21.3(0.9)$ \\
\hline & & 75 & $10.2(0.6)$ & $16.8(0.9)$ & - & - & $17.6(0.9)$ \\
\hline \multirow[t]{8}{*}{ Ischemic stroke } & Men & 45 & $0.9(0.2)$ & $3.7(0.4)$ & $7.9(0.7)$ & $12.6(1.1)$ & $13.9(1.4)$ \\
\hline & & 55 & $3.6(0.4)$ & $8.0(0.6)$ & $12.6(0.8)$ & $14.1(0.9)$ & $14.2(0.9)$ \\
\hline & & 65 & $5.4(0.5)$ & $10.1(0.7)$ & $11.9(0.8)$ & - & $11.9(0.8)$ \\
\hline & & 75 & $7.3(0.7)$ & $10.4(0.9)$ & - & - & $10.7(0.9)$ \\
\hline & Women & 45 & $0.4(0.1)$ & $1.6(0.2)$ & $4.7(0.4)$ & $9.4(0.8)$ & $10.6(1.0)$ \\
\hline & & 55 & $1.1(0.2)$ & $4.3(0.3)$ & $9.9(0.6)$ & $13.8(0.9)$ & $14.4(1.0)$ \\
\hline & & 65 & $2.8(0.3)$ & $8.4(0.5)$ & $12.6(0.7)$ & - & $13.5(0.8)$ \\
\hline & & 75 & $8.6(0.5)$ & $10.3(0.7)$ & - & - & $11.0(0.8)$ \\
\hline \multirow[t]{8}{*}{ Hemorrhagic stroke } & Men & 45 & $0.6(0.2)$ & $2.0(0.3)$ & $3.3(0.5)$ & $4.1(0.6)$ & $4.6(0.6)$ \\
\hline & & 55 & $1.7(0.3)$ & $3.2(0.4)$ & $4.0(0.5)$ & $4.5(0.5)$ & $4.5(0.5)$ \\
\hline & & 65 & $1.9(0.3)$ & $3.1(0.4)$ & $3.7(0.4)$ & - & $3.8(0.5)$ \\
\hline & & 75 & $2.3(0.3)$ & $3.5(0.5)$ & - & - & $3.5(0.5)$ \\
\hline & Women & 45 & $0.4(0.1)$ & $1.4(0.2)$ & $2.6(0.3)$ & $5.1(0.6)$ & $5.7(0.8)$ \\
\hline & & 55 & $0.9(0.1)$ & $2.4(0.2)$ & $4.3(0.4)$ & $5.5(0.5)$ & $5.5(0.5)$ \\
\hline & & 65 & $1.8(0.2)$ & $4.4(0.4)$ & $5.4(0.5)$ & - & $5.6(0.5)$ \\
\hline & & 75 & $3.3(0.4)$ & $4.8(0.5)$ & - & - & $4.9(0.5)$ \\
\hline
\end{tabular}

Abbreviation: LTR, lifetime risk.

of baseline age $(P=0.02)$, but when separated into stroke subtype, none was statistically significant. In contrast, we found that the LTR of all-stroke was not significantly different by time period in women $(P=0.21)$, but when data were assessed by stroke subtype, there were significant differences in the risk for ischemic stroke $(P=0.006)$.

\section{LTRs of all-stroke by BP group}

The JNC 7 BP group at index age exam was significantly associated with the LTR of all-stroke for men and women $(P<0.001$; Table 3$)$. Stages 1 and 2 hypertension status at baseline resulted in significantly higher risk of all-stroke in both men and women. In the stroke 
Table 3 Sex-specific short-, intermediate-term and LTR estimates for stroke and its subtypes beyond 55 years of age by BP groups: adult health study, 45-year follow-up, 1958-2003, Japan

\begin{tabular}{|c|c|c|c|c|c|c|c|}
\hline \multirow[b]{2}{*}{ Stroke type } & \multirow[b]{2}{*}{ Sex } & \multirow[b]{2}{*}{ BP group ${ }^{\mathrm{a}}$} & \multicolumn{4}{|c|}{ Short- and intermediate-term risk estimate (s.d.) (\%) } & \multirow{2}{*}{$\begin{array}{l}\text { LTR estimate } \\
\text { (s.d.) (\%) }\end{array}$} \\
\hline & & & 10 year & 20 year & 30 year & 40 year & \\
\hline \multirow[t]{8}{*}{ All-stroke } & Men & Normal & $2.6(0.6)$ & $7.8(1.3)$ & $13.1(1.9)$ & - & $13.8(2.0)$ \\
\hline & & PreHT & $3.3(0.6)$ & $8.4(1.1)$ & $14.1(1.5)$ & $16.9(1.7)$ & $16.9(1.7)$ \\
\hline & & Stage $1 \mathrm{HT}$ & $6.1(0.9)$ & $14.3(1.5)$ & $23.1(2.2)$ & $25.8(2.4)$ & $25.8(2.4)$ \\
\hline & & Stage 2 HT & $13.1(1.5)$ & $21.3(1.9)$ & $25.3(2.2)$ & - & $25.8(2.3)$ \\
\hline & Women & Normal & $1.2(0.3)$ & $5.3(0.7)$ & $11.3(1.3)$ & $16.0(1.7)$ & $16.0(1.7)$ \\
\hline & & PreHT & $1.1(0.3)$ & $5.0(0.6)$ & $12.6(1.2)$ & $19.4(1.8)$ & $19.9(1.8)$ \\
\hline & & Stage $1 \mathrm{HT}$ & $2.8(0.5)$ & $9.3(1.0)$ & $20.0(1.8)$ & $24.0(2.3)$ & $24.0(2.3)$ \\
\hline & & Stage $2 \mathrm{HT}$ & $5.5(0.8)$ & $15.7(1.4)$ & $26.4(2.1)$ & $30.5(2.4)$ & $30.5(2.4)$ \\
\hline \multirow[t]{8}{*}{ Ischemic stroke } & Men & Normal & $1.6(0.6)$ & $6.6(1.2)$ & $10.6(1.7)$ & - & $11.2(1.8)$ \\
\hline & & PreHT & $1.8(1.7)$ & $4.7(2.6)$ & $8.7(1.2)$ & $10.7(1.5)$ & $10.7(1.5)$ \\
\hline & & Stage $1 \mathrm{HT}$ & $3.6(0.7)$ & $9.2(1.3)$ & $16.2(1.9)$ & $18.3(2.2)$ & $18.3(2.2)$ \\
\hline & & Stage $2 \mathrm{HT}$ & $8.5(1.3)$ & $13.5(1.6)$ & $16.8(1.9)$ & - & $17.3(2.0)$ \\
\hline & Women & Normal & $1.0(0.3)$ & $3.2(0.6)$ & $7.7(1.1)$ & $11.3(1.5)$ & $11.3(1.5)$ \\
\hline & & PreHT & $0.5(0.2)$ & $2.3(0.4)$ & $7.2(1.0)$ & $12.6(1.5)$ & $13.1(1.6)$ \\
\hline & & Stage $1 \mathrm{HT}$ & $1.3(0.4)$ & $5.2(0.8)$ & $11.7(1.5)$ & $14.5(2.0)$ & $14.5(2.0)$ \\
\hline & & Stage $2 \mathrm{HT}$ & $2.2(0.5)$ & $8.6(1.1)$ & $16.2(1.8)$ & $18.4(2.0)$ & $18.4(2.0)$ \\
\hline \multirow[t]{8}{*}{ Hemorrhagic stroke } & Men & Normal & $0.6(0.3)$ & $0.6(0.3)$ & $1.5(0.7)$ & - & $1.5(0.7)$ \\
\hline & & PreHT & $1.0(0.3)$ & $2.6(0.6)$ & $3.5(0.8)$ & $4.2(0.9)$ & $4.2(0.9)$ \\
\hline & & Stage $1 \mathrm{HT}$ & $2.2(0.6)$ & $4.1(0.9)$ & $4.8(1.0)$ & $5.4(1.2)$ & $5.4(1.2)$ \\
\hline & & Stage 2 HT & $3.7(0.8)$ & $6.0(1.1)$ & $6.4(1.2)$ & - & $6.4(1.2)$ \\
\hline & Women & Normal & $0.1(0.08)$ & $1.3(0.4)$ & $2.6(0.7)$ & $3.7(0.9)$ & $3.7(0.9)$ \\
\hline & & PreHT & $0.4(0.17)$ & $1.6(0.3)$ & $3.6(0.7)$ & $4.7(0.8)$ & $4.7(0.8)$ \\
\hline & & Stage $1 \mathrm{HT}$ & $1.3(0.3)$ & $2.9(0.6)$ & $5.3(0.9)$ & $6.2(1.1)$ & $6.2(1.1)$ \\
\hline & & Stage $2 \mathrm{HT}$ & $2.6(0.6)$ & $5.5(0.9)$ & $6.9(1.0)$ & $8.9(1.4)$ & $8.9(1.4)$ \\
\hline
\end{tabular}

Abbreviations: BP, blood pressure; DBP, diastolic BP; HT, hypertension; JNC 7, Seventh Report of the Joint National Committee on the Prevention, Detection, Evaluation, and Treatment of High Blood Pressure; LTR, lifetime risk; SBP, systolic BP.

${ }^{a}$ BP groups were defined using SBP and DBP, and followed criteria from the JNC 7 classification: ${ }^{12}$ normal, SBP $<120 \mathrm{~mm} \mathrm{Hg}$ and DBP $<80 \mathrm{~mm} \mathrm{Hg}$; preHT, SBP $120-139 \mathrm{~mm} \mathrm{Hg}$ and/or DBP 80-89 mm Hg; stage $1 \mathrm{HT}$, SBP $140-159 \mathrm{~mm} \mathrm{Hg}$ and/or DBP 90-99 mm Hg; and stage $2 \mathrm{HT}$, SBP $\geqslant 160 \mathrm{~mm} \mathrm{Hg}$ and/or DBP $\geqslant 100 \mathrm{~mm} \mathrm{Hg}$.

subtype-specific analyses, there were significant differences in risk between men and women for hemorrhagic stroke $(P<0.001$ between each BP group) and ischemic stroke $(P<0.001$ between each BP group). Similar results were also seen for the other index age cohorts.

\section{DISCUSSION}

Our findings showed the mortality-adjusted residual LTR of stroke beyond 55 years of age was one in five involving a sample of 7487 middle-aged Japanese who have been followed up for 45 years; female stroke LTR was slightly higher compared with male LTR $(20.5 \%$ for men vs. $22.2 \%$ for women). Separated into stroke subtypes, the observed probabilities were higher for ischemic stroke (one in seven for men and women) than for hemorrhagic stroke (one in 20 for both sexes). The LTR for all-stroke and stroke subtypes were similar across the other index ages. In addition, we stratified subjects into groups who turned 55 years of age in the first (1958-1977), second (19781990) or third periods (1991+), because our study observation period corresponded to rapid westernization in Japan and drastic changes in risk-factor profiles due to widespread use of antihypertensive drug therapy. ${ }^{18}$ We found differences in risk for stroke and stroke subtype by gender and time group, which may support a past report indicating that risks of any stroke subtype have leveled off during the last few decades. ${ }^{19}$ However, we were unable to draw conclusions on different risks by time group because of the inadequate number of cases available for analysis, especially for men.
A few reports have been published on the LTR of stroke from the Rotterdam Study (6-year follow-up), ${ }^{5}$ the Framingham Study (>50-year follow-up $)^{4}$ and the Suita Study (17-year follow-up); ${ }^{6}$ and the LTR of stroke in our study was similar to the reported results from the these studies (about 20\% for both men and women of 55 years of age). Male gender has been considered an important risk factor for stroke, ${ }^{20}$ but the higher LTR in women of 55 years of age in the AHS has been attributed to the longer life span of women. There may be, however, other potential reasons that women are at greater risk than men for atrial fibrillation-related thromboembolic events. $^{21,22}$ Elderly women (above age 85 years) were observed to have higher stroke incidence than that in men. ${ }^{23-25}$ Our LTR estimates emphasized that the LTR remains a major threat and public health burden for Japan's aging society, especially for women.

Our longitudinal study using LTR estimates indicated that hypertension in midlife (40s, 50s) remained a risk factor for stroke compared with normotensive subjects, which supports a past report indicating that elevated BP in the 40s is suggested to have the so-called 'carryover' effects on stroke incidence for subjects when they reach their 60 s. $^{26}$ Moreover, our findings suggested that stroke risk of subjects with prehypertension in their 50 s, but not for such subjects in their 40s, was similar to that of normotensive subjects. Recent studies about relationship between elevated BP and PAF for stroke incidence of Japanese in the Japan Public Health Centre study ${ }^{27}$ and the Circulatory Risk in Communities Study ${ }^{28}$ suggested that the 
increase in population attributable fraction (PAF) when hypertension progressed from high normal to mild becomes more pronounced, indicating that BP levels lower than $140 / 90 \mathrm{~mm} \mathrm{Hg}$ may not necessarily represent stroke risk, which were supportive for us. On the other hand, Kokubo et al. reported significantly elevated risk of stroke according to BP category comparing normal and high-normal BPs in a general urban Japanese men. ${ }^{29}$ Those controversial issues regarding prehypertension impact on stroke events could be because of white coat hypertension and the potential impact of fluctuations in BP levels after midlife that may affect stroke incidence. Further analysis of the relation between BP fluctuation and stroke could shed light on the issue whether early BP control over normal levels at midlife could be beneficial for prevention of stroke incidence.

Our study has several strengths, including a large population not preselected for existing disease or occupational fitness, a 45-year follow-up with biennial health examinations and virtually complete mortality ascertainment. In addition, we believe medical surveillance bias to be minimal, as the entire cohort is eligible for free, special medical care. Our estimates were based on simultaneously gathered data on both stroke incidence and other-cause mortality attributable to the competing risk of death in the same cohort. During recent decades in Japan, ischemic stroke has been reported to be the dominant subtype as a proportion of all strokes, being three to four times more frequent than cerebral hemorrhage, which is similar to our findings. ${ }^{30,31}$

The study also has several limitations and uncertainties. The possible stroke cases with stroke-related ICD codes were reviewed retrospectively. Ascertainment of stroke events from death certificates or cases before the widespread use of imaging tests (beginning around the 1970s) is of limited diagnostic accuracy and represents only a fraction of cases of incident disease. We did not consider the BP-lowering effects of antihypertensive agents because of unavailable medication information before 1990. Finally, atomic bomb survivors exposed to radiation were included in this analysis, and thus our results may not accurately reflect the general population.

In conclusion, our study suggests that hypertension at midlife among Japanese subjects confers a LTR for stroke. It is important to address this risk early when hypertension is identified to ensure that proper therapy can be initiated and stroke risk lowered.

\section{ACKNOWLEDGEMENTS}

The RERF, Hiroshima and Nagasaki, Japan is a private, non-profit foundation funded by the Japanese Ministry of Health, Labour and Welfare, and the United States Department of Energy, the latter, in part, through the National Academy of Sciences. This publication was supported by the RERF Research Protocol RP\# 2-75.

1 Bonita R, Stewart A, Beaglehole R. International trends in stroke mortality: 1970-1985. Stroke 1990; 21: 989-992.

2 Kubo M, Kiyohara Y, Kato I, Tanizaki Y, Arima H, Tanaka K, Nakamura H, Okubo K, lida $M$. Trends in the incidence, mortality, and survival rate of cardiovascular disease in a Japanese community: the Hisayama study. Stroke 2003; 34: 2349-2354.

3 Lewington S, Clarke R, Qizilbash N, Peto R, Collins R. Age-specific relevance of usual blood pressure to vascular mortality: a meta-analysis of individual data for one million adults in 61 prospective studies. Lancet 2002; 360: 1903-1913.

4 Seshadri S, Wolf PA. Lifetime risk of stroke and dementia: current concepts, and estimates from the Framingham Study. Lancet Neurol 2007; 6: 1106-1114.

5 Hollander M, Koudstaal PJ, Bots ML, Grobbee DE, Hofman A, Breteler MM. Incidence, risk, and case fatality of first ever stroke in the elderly population. The Rotterdam Study. J Neurol Neurosurg Psychiatry 2003; 74: 317-321.

6 Turin TC, Kokubo Y, Murakami Y, Higashiyama A, Rumana N, Watanabe M, Okamura T. Lifetime risk of stroke in Japan. Stroke 2010; 41: 1552-1554.
7 Pierce DA, Shimizu Y, Preston DL, Vaeth M, Mabuchi K. Studies of the mortality of atomic bomb survivors. Report 12, Part I. Cancer: 1950-1990. Radiat Res 1996; 146: $1-27$.

8 Shimizu Y, Pierce DA, Preston DL, Mabuchi K. Studies of the mortality of atomic bomb survivors. Report 12, part II. Noncancer mortality: 1950-1990. Radiat Res 1999; 152: 374-389.

9 Asplund K, Bonita R, Kuulasmaa K, Rajakangas AM, Schaedlich H, Suzuki K, Thorvaldsen $\mathrm{P}$, Tuomilehto J. Multinational comparisons of stroke epidemiology. Evaluation of case ascertainment in the WHO MONICA Stroke Study. World Health Organization Monitoring Trends and Determinants in Cardiovascular Disease. Stroke 1995; 26: 355-360.

10 Thorvaldsen P, Asplund K, Kuulasmaa K, Rajakangas AM, Schroll M. Stroke incidence, case fatality, and mortality in the WHO MONICA project. World Health Organization Monitoring Trends and Determinants in Cardiovascular Disease. Stroke 1995; 26: 361-367.

11 Takeya Y, Popper JS, Shimizu Y, Kato H, Rhoads GG, Kagan A. Epidemiologic studies of coronary heart disease and stroke in Japanese men living in Japan, Hawaii and California: incidence of stroke in Japan and Hawaii. Stroke 1984; 15: 15-23.

12 Chobanian AV, Bakris GL, Black HR, Cushman WC, Green LA, Izzo Jr JL, Jones DW, Materson BJ, Oparil S, Wright Jr JT, Roccella EJ. The seventh report of the joint national committee on prevention, detection, evaluation, and treatment of high blood pressure: the JNC 7 report. JAMA 2003; 289: 2560-2572.

13 Gray B. cmprsk: Subdistribution Analysis of Competing Risks. R package version 2.2-1. http://CRAN.R-project.org/package=cmprsk, 2010.

14 Pintilie M. Competing Risks: A Practical Perspective. John Wiley \& Sons: West Sussex, England, 2006.

15 Scrucca L, Santucci A, Aversa F. Competing risk analysis using R: an easy guide for clinicians. Bone Marrow Transplant 2007; 40: 381-387.

$16 \mathrm{Kim} \mathrm{HT}$. Cumulative incidence in competing risks data and competing risks regression analysis. Clin Cancer Res 2007; 13: 559-565.

17 Carandang R, Seshadri S, Beiser A, Kelly-Hayes M, Kase CS, Kannel WB, Wolf PA. Trends in incidence, lifetime risk, severity, and 30-day mortality of stroke over the past 50 years. JAMA 2006; 296: 2939-2946.

18 Kubo M, Hata J, Doi Y, Tanizaki Y, lida M, Kiyohara Y. Secular trends in the incidence of and risk factors for ischemic stroke and its subtypes in Japanese population. Circulation 2008; 118: 2672-2678.

19 Kita Y, Turin TC, Ichikawa M, Sugihara H, Morita Y, Tomioka N, Rumana N, Okayama A, Nakamura Y, Abbott RD, Ueshima H. Trend of stroke incidence in a Japanese population: Takashima stroke registry, 1990-2001. Int J Stroke 2009; 4: 241-249.

20 Boysen G, Nyboe J, Appleyard M, Sorensen PS, Boas J, Somnier F, Jensen G, Schnohr P. Stroke incidence and risk factors for stroke in Copenhagen, Denmark. Stroke 1988; 19: 1345-1353.

21 Fang MC, Singer DE, Chang Y, Hylek EM, Henault LE, Jensvold NG, Go AS. Gender differences in the risk of ischemic stroke and peripheral embolism in atrial fibrillation: the AnTicoagulation and risk factors in atrial fibrillation (ATRIA) study. Circulation 2005; 112: 1687-1691.

22 Wang TJ, Massaro JM, Levy D, Vasan RS, Wolf PA, D’Agostino RB, Larson MG, Kannel WB, Benjamin EJ. A risk score for predicting stroke or death in individuals with new-onset atrial fibrillation in the community: the Framingham Heart Study. JAMA 2003; 290: 1049-1056.

23 Glader EL, Stegmayr B, Norrving B, Terent A, Hulter-Asberg K, Wester PO, Asplund K. Sex differences in management and outcome after stroke: a Swedish national perspective. Stroke 2003; 34: 1970-1975.

24 Rothwell PM, Coull AJ, Silver LE, Fairhead JF, Giles MF, Lovelock CE, Redgrave JN, Bull LM, Welch SJ, Cuthbertson FC, Binney LE, Gutnikov SA, Anslow P, Banning AP, Mant D, Mehta Z. Population-based study of event-rate, incidence, case fatality, and mortality for all acute vascular events in all arterial territories (Oxford Vascular Study). Lancet 2005; 366: 1773-1783.

25 Petrea RE, Beiser AS, Seshadri S, Kelly-Hayes M, Kase CS, Wolf PA. Gender differences in stroke incidence and poststroke disability in the Framingham heart study. Stroke 2009; 40: 1032-1037.

26 Seshadri S, Wolf PA, Beiser A, Vasan RS, Wilson PW, Kase CS, Kelly-Hayes M, Kannel WB, D'Agostino RB. Elevated midlife blood pressure increases stroke risk in elderly persons: the Framingham Study. Arch Intern Med 2001; 161: 2343-2350.

27 Ikeda A, Iso H, Yamagishi K, Inoue M, Tsugane S. Blood pressure and the risk of stroke, cardiovascular disease, and all-cause mortality among Japanese: the JPHC Study. Am J Hypertens 2009; 22: 273-280.

28 Imano H, Kitamura A, Sato S, Kiyama M, Ohira T, Yamagishi K, Noda H, Tanigawa T, Iso $\mathrm{H}$, Shimamoto $\mathrm{T}$. Trends for blood pressure and its contribution to stroke incidence in the middle-aged Japanese population: the Circulatory Risk in Communities Study (CIRCS). Stroke 2009; 40: 1571-1577.

29 Kokubo Y, Kamide K, Okamura T, Watanabe M, Higashiyama A, Kawanishi K, Okayama A, Kawano Y. Impact of high-normal blood pressure on the risk of cardiovascular disease in a Japanese urban cohort: the Suita study. Hypertension 2008; 52: 652-659.

30 Ishikawa S, Kayaba K, Gotoh T, Nago N, Nakamura Y, Tsutsumi A, Kajii E. Incidence of total stroke, stroke subtypes, and myocardial infarction in the Japanese population: the JMS Cohort Study. J Epidemiol 2008; 18: 144-150.

31 Kita Y, Okayama A, Ueshima H, Wada M, Nozaki A, Choudhury SR, Bonita R, Inamoto Y, Kasamatsu T. Stroke incidence and case fatality in Shiga, Japan 1989-1993. Int J Epidemiol 1999; 28: 1059-1065. 